\title{
A functionalized graphene aerogel for efficient water purification
}

Chuan Cao MSc

Key Laboratory of Coal Science and Technology of Ministry of Education and Shanxi Province, Taiyuan University of Technology, Taiyuan, China

Jing Ren $\mathrm{PhD}$

Key Laboratory of Coal Science and Technology of Ministry of Education and Shanxi Province, Taiyuan University of Technology, Taiyuan, China

\author{
Yong-Kang Lv PhD \\ Key Laboratory of Coal Science and Technology of Ministry of Education \\ and Shanxi Province, Taiyuan University of Technology, Taiyuan, China \\ Rui-Peng Ren PhD \\ Key Laboratory of Coal Science and Technology of Ministry of Education \\ and Shanxi Province, Taiyuan University of Technology, Taiyuan, China \\ (corresponding author: renruipeng@tyut.edu.cn)
}

\begin{abstract}
Water pollution has become a worldwide problem due to ever-increasing industrial wastewater. An ideal adsorbing material is able to remove oils, organic solvents and dyes from water efficiently, particularly with a high adsorption capacity and reusability. Herein, the authors report a novel strategy of synthesis of a three-dimensional functionalized graphene aerogel (FGA) with porous structures for effective water purification. The FGA is prepared by a facile one-step hydrothermal method, in which graphene oxide is reduced by ethylenediamine and modified by dodecyltrimethoxysilane to enhance hydrophobicity. When used as an adsorbing material, the FGA exhibits a high adsorption capacity (130-242 g/g for oils and organic solvents, 197-596 mg/g for dyes). Furthermore, the FGA also exhibits high reusability and durability by being reused for organic solvent-water separation for ten cycles without losing its hydrophobicity, which makes it a good candidate for industrial water treatment.
\end{abstract}

\section{Notation}

$c_{0} \quad$ initial concentration of the dye

$c_{1} \quad$ final concentration of the dye

$M \quad$ weight after the functionalized graphene aerogel (FGA)

has adsorbed the organic solvent

$M_{0} \quad$ weight of FGA

$m$ weight of FGA

$Q_{\mathrm{e}} \quad$ dye adsorption capacity of FGA

$Q_{\mathrm{w}} \quad$ adsorption capacity of FGA

$V \quad$ volume of the aqueous dye solution

\section{Introduction}

With the rapid development of industrialization, environmental pollution problems have attracted more and more attention. For example, oil spillage and chemical leakage accidents have occurred frequently during exploration and transportation processes, which result in severe environmental pollution. ${ }^{1-3}$ Therefore, many processes, such as chemical precipitation, ${ }^{4}$ physical adsorption, ${ }^{5,6}$ biodegradation method $^{7,8}$ and membrane separation, ${ }^{9-12}$ have been developed to address oil spill pollution. Among these methods, the use of adsorption materials is considered one of the most promising methods for the removal of oils and organic solvents from water. Although traditional adsorption materials, including activated carbon (C), ${ }^{13}$ wool fibers, ${ }^{14}$ zeolites, ${ }^{15}$ straw ${ }^{16}$ and fly ash, ${ }^{17}$ have been widely used to remove oil from water, they still suffer from low adsorption capacities and poor recyclability. Thus, it is still a challenge to develop adsorption materials with high adsorption capacity and reusability.

Graphene, a novel two-dimensional (2D) material with remarkable properties, ${ }^{18-20}$ has attracted more and more attention due to its remarkable properties and is used in many fields, such as sensors, ${ }^{21,22}$ supercapacitors, ${ }^{23}$ catalysts $^{24,25}$ and so on. ${ }^{26}$ To translate the outstanding properties of $2 \mathrm{D}$ graphene into a macroscopic perspective, graphene should be assembled into a three-dimensional (3D) porous monolithic material. There are several methods for preparing 3D aerogels, including one-step hydrothermal method, ${ }^{27}$ chemical vapor deposition, ${ }^{28,29}$ bidirectional freeze-drying approach $^{30}$ and 3D printing. $^{31}$ Typically, these graphene aerogels (GAs) show a porous structure, a high specific surface area and hydrophobicity, which means that they can be used as adsorbent materials for water purification. Chen et $a l .{ }^{32}$ reported a GA prepared by hydrothermal treatment and the freeze-drying method for oil absorption; its absorption capacity could reach 224-522 times its own weight. Hong et al. ${ }^{33}$ prepared functionalized GA (FGA) by surface modification of GAs for adsorption of oils and organic solvents. Compared with the water contact angle (WCA) of the pristine GA $\left(103^{\circ}\right)$, the WCA of the functionalized GA was higher $\left(144^{\circ}\right)$. Benefitting from the structural features and hydrophobic surface properties, the FGA exhibited excellent absorption performance for oils and organic solvents (capacity up to 112 times its weight). In addition to oils and organic solvents, GAs have also been used to adsorb organic dyes. Chen et al. ${ }^{34}$ prepared GA using the small organic molecule of cysteamine as a covalent cross-linker. The resultant graphene/cysteamine aerogels exhibited a high adsorption capacity for dyes, such as methylene blue $(207.8 \mathrm{mg} / \mathrm{g})$ and methyl orange (MO) $(70.2 \mathrm{mg} / \mathrm{g})$. Therefore, the GAs with high specific surface areas, high adsorption capacities and hydrophobic surface properties have great potential as recyclable and durable adsorbent materials for water purification.

Here, the authors report an FGA as an efficient and recyclable absorbent for the removal of oils, organic solvents and dyes from water. Graphene oxide (GO) sheets with numerous oxygen 
(O)-containing groups can link with ethylenediamine (EDA) and dodecyltrimethoxysilane (DTMS) through chemical reactions and realize the functionalization of graphene sheets. Benefitting from the hydrophobic nature of DTMS and the porous network of GAs, the FGA exhibits excellent hydrophobicity. The FGA can absorb a wide range of oils and organic solvents with an adsorption capacity of 130-242 g/g. More importantly, the FGA also exhibits excellent recyclability and maintains a high adsorption capacity even after ten cycles through distillation. The authors believe that the FGA with good adsorption performance will have great potential for water purification.

\section{Results and discussion}

The synthesis process of FGA is illustrated schematically in Figure 1. Firstly, a weak reducing agent (EDA) was introduced into the GO solution to functionalize it and initiate GO sheet assembly into a 3D network. At the same time, DTMS was used to modify the GO sheets, thus improving hydrophobicity. Secondly, after the hydrothermal treatment, the resultant functionalized graphene hydrogel (FGH) was obtained, which exhibited negligible volume shrinkage. Finally, the solvent was removed from the graphene hydrogel by freeze-drying to produce FGA. The reaction mechanism of the DTMS modification is shown in Figure S1 in the online supplementary material. DTMS is hydrolyzed under alkaline conditions, which the alkoxy group is converted to silanol groups. ${ }^{35}$ Then, the silanol groups react with the hydroxyl group on the GO sheets to form covalent bonds under heating; meanwhile, water molecules are eliminated. ${ }^{36}$

The interconnected porous structure of FGA with a pore size of several to dozens of micrometers was observed by scanning electron microscopy (SEM) (Figures 2(a) and 2(b)), where the pore walls consisted of partially stacked graphene sheets. In contrast, conventional GA made by the high-temperature hydrothermal method always has thicker pore walls, which are composed of severely stacked graphene sheets (Figure S2 in the online supplementary material). The SEM results indicate that EDA functionalization can suppress the restacking of graphene sheets during assembly, which can effectively improve the specific surface area of FGA. Meanwhile, compared with the smooth surface of pure graphene sheets (Figure S3 in the online supplementary material), the DTMS-modified graphene sheets show increased roughness (Figure 2(c)), which is beneficial for

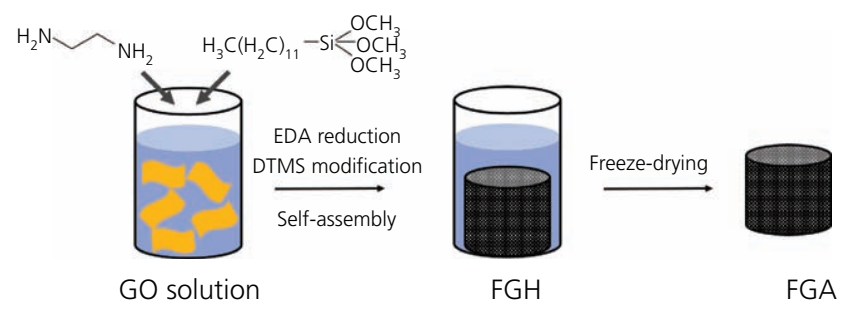

Figure 1. Illustration of the fabrication process of the FGA hydrophobicity. The wetting properties of FGA were studied by WCA measurement. When comparing the images in Figure 2(d), the DTMS-modified FGA shows a higher WCA of nearly $135^{\circ}$ than that of pure $\mathrm{GA}\left(\mathrm{WCA}=106^{\circ}\right)$, which confirms that DTMS modification can enhance hydrophobicity. Thus, the hydrophobic FGA with a porous structure can be used as adsorbing material. The energy-dispersive X-ray spectroscopy mapping of silicon and nitrogen is shown in Figures 2(e) and 2(f), which confirm that DTMS and EDA appear on the surface of GA.

The specific surface area is an important factor for the adsorbing material. Therefore, the specific surface area of the as-prepared FGA was characterized by nitrogen adsorption-desorption at $77 \mathrm{~K}$, and the result is shown in Figure 3(a). The specific surface area calculated from the Brunauer-Emmett-Teller $\operatorname{model}^{37}$ is $141.4 \mathrm{~m}^{2} / \mathrm{g}$ for FGA. The total pore volume is $0.441 \mathrm{~cm}^{3} / \mathrm{g}$ by calculation. The high specific surface area of FGA is advantageous in oil adsorption. In addition, Fourier transform infrared spectroscopy (FT-IR), Raman spectroscopy and X-ray diffraction (XRD) were conducted to reveal microstructural evolution. FT-IR analysis was further carried out to investigate the variation of the oxygen-containing functional groups in the reaction process. As shown in Figure 3(b), there are numerous oxygen-containing functional groups on $\mathrm{GO}$, including the peak at $3424 \mathrm{~cm}^{-1}$ for $-\mathrm{OH}$ (stretching vibration), ${ }^{38}$ at $1733 \mathrm{~cm}^{-1}$ for $\mathrm{C}=\mathrm{O}$ (stretching vibration), at $1623 \mathrm{~cm}^{-1}$ for $\mathrm{C}=\mathrm{C}$, at $1418 \mathrm{~cm}^{-1}$ for carboxy $\mathrm{C}-\mathrm{O}$,
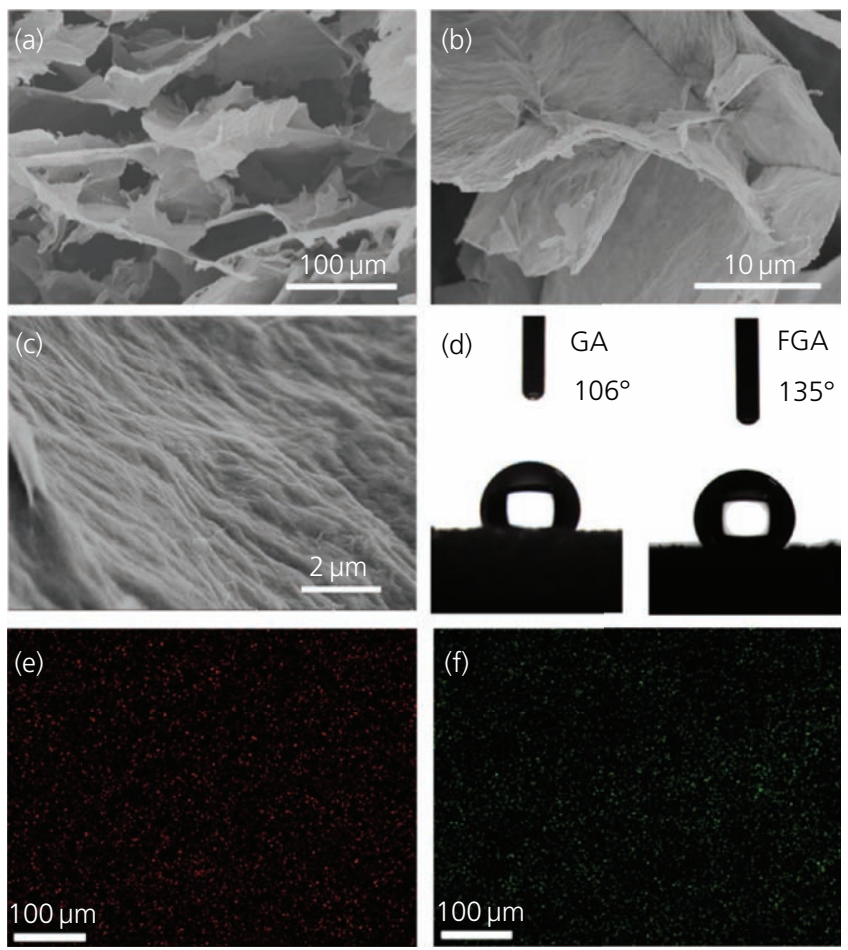

Figure 2. (a-c) SEM images of FGA; (d) measurement WCAs of pure $G A$ and $F G A ;(e, f)$ elemental mapping showing the presence of silicon and nitrogen on the GA surface 


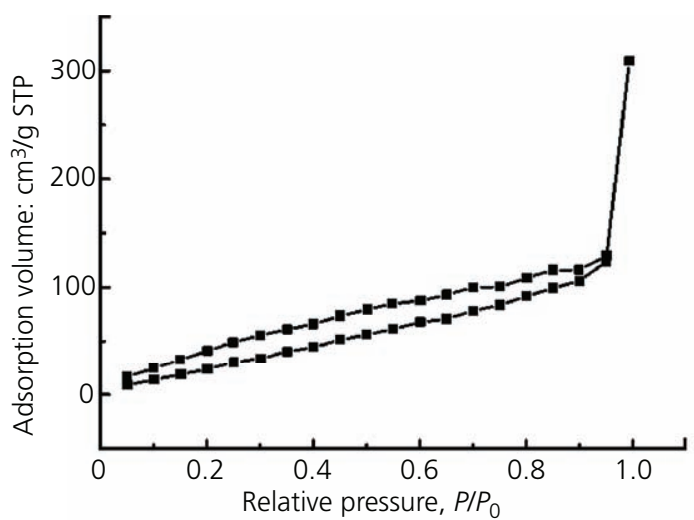

(a)

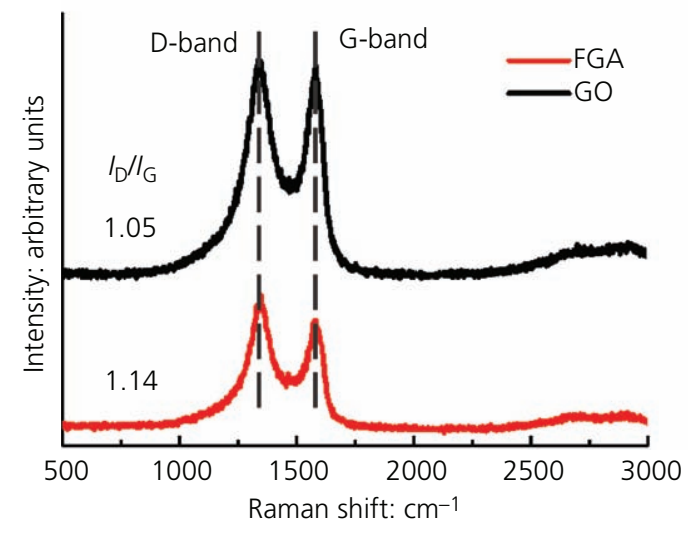

(c)

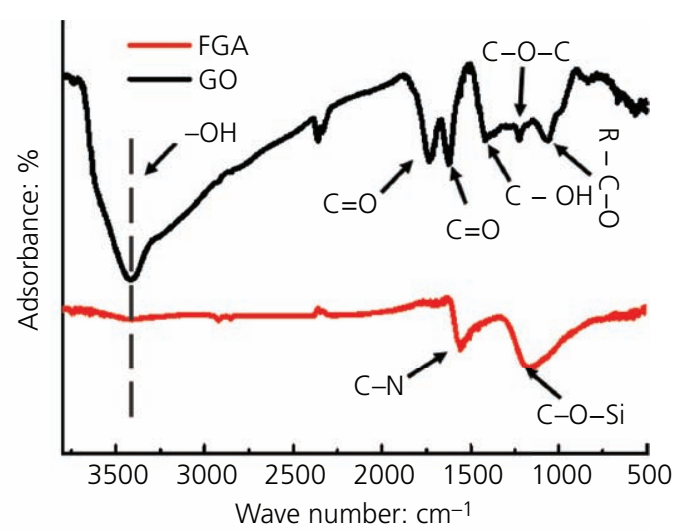

(b)

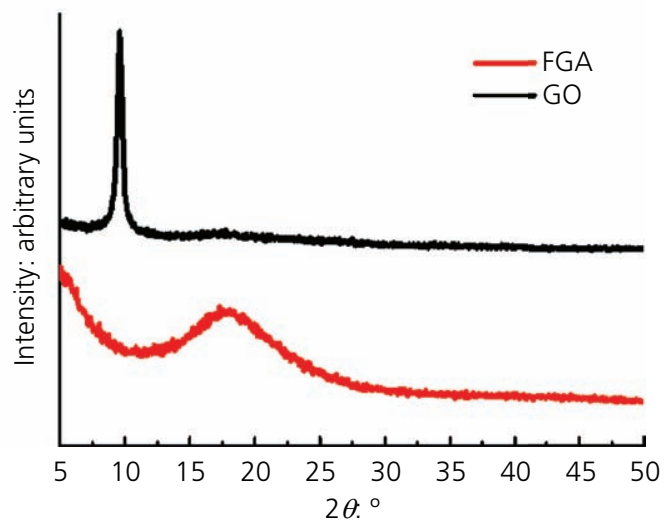

(d)

Figure 3. (a) Nitrogen adsorption-desorption isotherm of FGA (STP, standard pressure and temperature); (b) Fourier transform infrared spectra; (c) Raman spectra; (d) X-ray diffraction patterns of GO and FGA

at $1225 \mathrm{~cm}^{-1}$ for epoxy $\mathrm{C}-\mathrm{O}$ and at $1054 \mathrm{~cm}^{-1}$ for alkoxy C-O. ${ }^{39}$ In comparison, FGA shows weak spectral intensities, and the peaks located at $1733,1418,1225$ and $1054 \mathrm{~cm}^{-1}$ almost disappeared. This indicates that GO was reduced by EDA during the hydrothermal process. Moreover, an additional peak appears at $1558 \mathrm{~cm}^{-1}$ for $\mathrm{C}-\mathrm{N},{ }^{40}$ which demonstrates that EDA is grafted onto $\mathrm{GO}^{41}$ Another new peak, appearing at $1088 \mathrm{~cm}^{-1}$ for $\mathrm{C}-\mathrm{O}-\mathrm{Si}$, indicates that DTMS reacts with the $-\mathrm{OH}$ of $\mathrm{GO}$ and forms a covalent bond to cross-link. ${ }^{36}$ The Raman spectrum shows two peaks located at nearly 1320 and $1590 \mathrm{~cm}^{-1}$, corresponding to the D- and G-bands (Figure 3(c)). The intensity ratio $\left(I_{\mathrm{D}} / I_{\mathrm{G}}\right)$ can be used to evaluate the degree of graphitization in the carbon samples. ${ }^{42}$ The $I_{\mathrm{D}} / I_{\mathrm{G}}$ ratio is calculated to be 1.05 and 1.14 for GO and FGA, respectively. The higher $I_{\mathrm{D}} / I_{\mathrm{G}}$ ratio of FGA can be attributed to the EDA and DTMS functional group, which introduces defects to graphene sheets. ${ }^{43}$ The graphitization degree of the as-prepared samples was also estimated by the using the XRD patterns. As seen in Figure 3(d), the XRD pattern of GO shows a sharp peak at $10.2^{\circ}$, which indicates that the crystallinity of GO is relatively high, ${ }^{44,45}$ corresponding to an interlayer space of $0.87 \mathrm{~nm}$, which can be attributed to the oxygen-containing functional groups on the GO sheets. As for the FGA, the interlayer space is calculated to be $0.50 \mathrm{~nm}$, which indicates that the oxygencontaining functional groups are removed. ${ }^{46,47}$

The variation of the elemental composition was determined by X-ray photoelectron spectrometry (XPS) analysis. As shown in Figure 4(a), the intensity of the peak corresponding to carbon located at $284.8 \mathrm{eV}^{48}$ increases from GO to FGA, while the change in the oxygen content (at $532 \mathrm{eV}$ ) shows the opposite trend. The XPS analysis further confirms the reduction of GO (the carbon/oxygen atomic ratio is 1.89) to FGA (the carbon/oxygen atomic ratio is 7.39) during the hydrothermal process. As shown in Figure 4(b), the presence of $\mathrm{C}-\mathrm{N}(285.4 \mathrm{eV})^{49}$ and $\mathrm{C}-\mathrm{O}-\mathrm{Si}$ $(285.0 \mathrm{eV})^{50}$ peaks in the high-resolution carbon $1 \mathrm{~s}$ spectrum of FGA suggests that EDA and DTMS were grafted onto graphene sheets successfully.

The 3D porous structure and hydrophobicity of the FGA make it an ideal candidate for the removal of oils and organic solvents. When an FGA was placed on the surface of organic solvent-water mixtures, the organic solvent layer (dyed with Sudan Red I) started shrinking and disappeared completely after a few minutes (Figure 5(a)). To determine further the adsorption ability of FGA, 


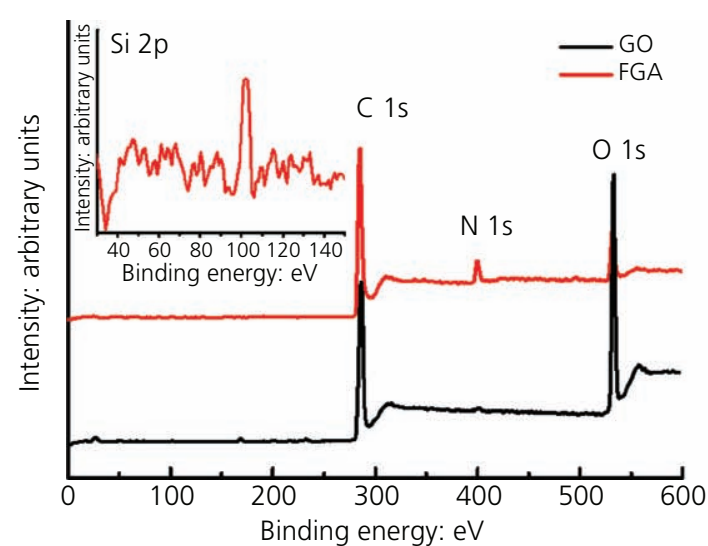

(a)

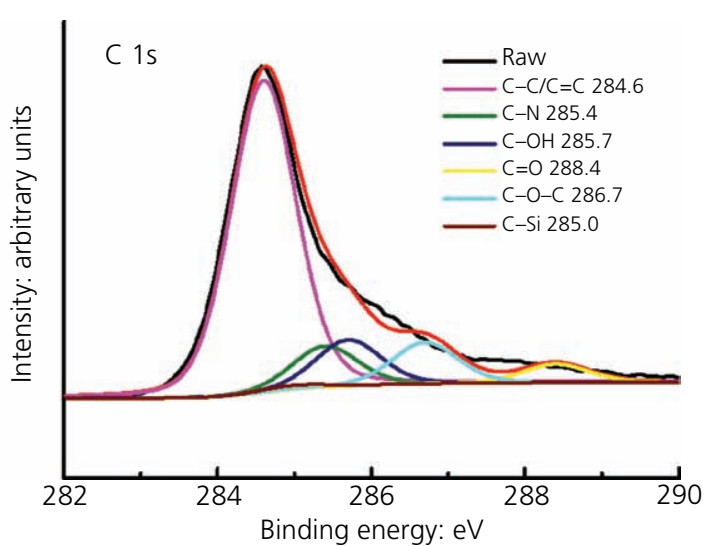

(b)

Figure 4. (a) XPS survey spectra of GO and FGA; (b) carbon 1s spectrum of FGA
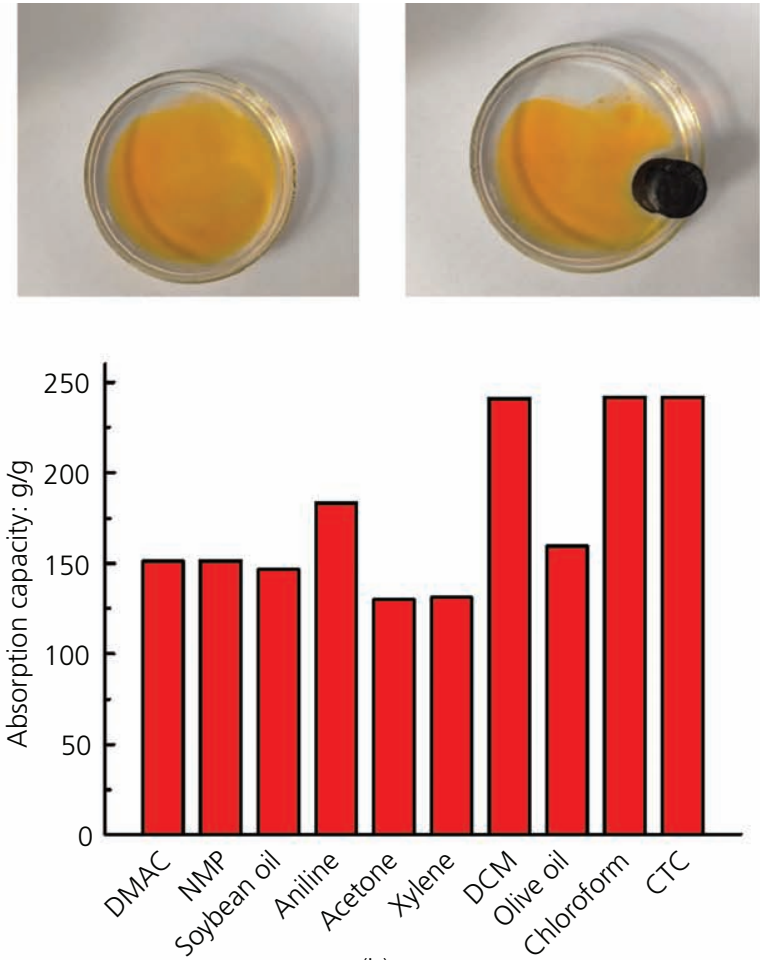

(b)
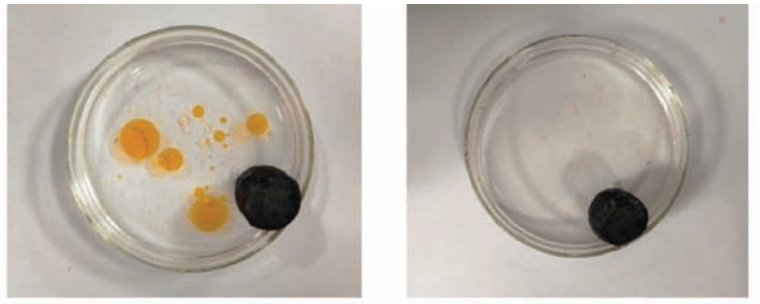

(a)

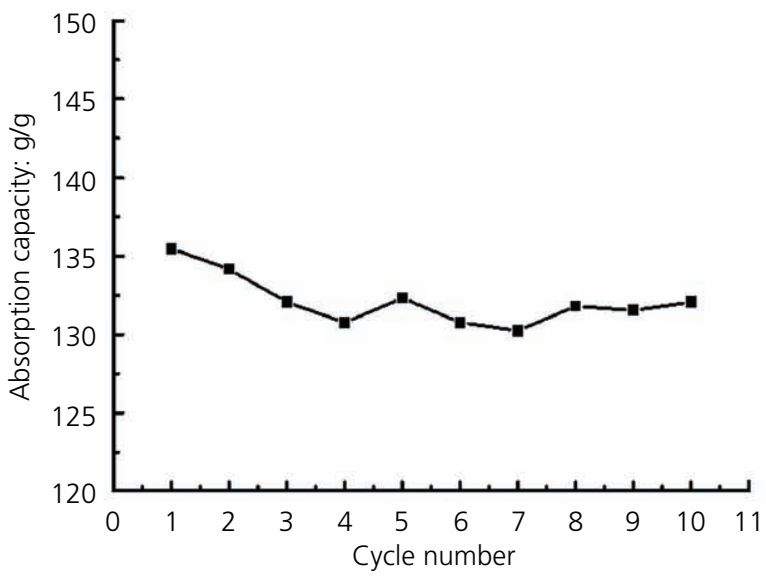

(c)

Figure 5. Adsorption tests of FGA: (a) adsorption process of xylene (stained with Sudan Red I) on the surface of water by the FGA; (b) adsorption capacities of FGA for various oils and organic solvents (DMAC, dimethylacetamide; NMP, N-methyl-2-pyrrolidone; DCM, dichloromethane; CTC, carbon tetrachloride); (c) adsorption recyclability of FGA over ten cycles

the authors investigated its adsorption capacities for different oils/ organic solvents. The FGA showed excellent adsorption capacities ranging from 130 to $242 \mathrm{~g} / \mathrm{g}$. Recyclability is a key requirement in practical applications. Therefore, the authors further evaluated the recyclability of FGA through a simple sorption-distillation cycle by taking xylene as an example (Figure 5(b)). The vapor of the xylene was collected for recovery, and the FGA was regenerated. The sorption-distillation process was repeated ten times to study the recyclability of FGA. As shown in Figure 5(c), the FGA showed stable sorption performance and no obvious decrease in sorption capacity was observed.

The adsorption mechanism for organic solvents and oils is as follows. There is a relationship between the adsorption of solids to liquids and their surface wettability. Therefore, the wettability of the adsorbent surface is important in oil-water separation. ${ }^{51}$ 
The wettability of a solid depends on its surface energy and morphology, and the wettability of a solid can be changed by adjusting these two factors. ${ }^{52}$ Therefore, the authors choose DTMS with low surface energy as the hydrophobic agent while grafting DTMS onto FGA to reduce surface energy. On the other hand, introduction of DTMS results in a rough surface of FGA. As a result, the FGA possesses hydrophobicity and lipophilicity. Therefore, FGA can adsorb organic solvents and oils from water.

In addition to oils and organic solvents, the FGA also shows high adsorption capability for dyes. As shown in Figures 6(a)-6(c), when FGA (1 mg) was put into aqueous rhodamine B (RB), MO and methyl blue (MB) solutions $(8 \mathrm{ml}, 20 \mathrm{mg} / \mathrm{l})$ for $12 \mathrm{~h}$, the color of each of the solutions gradually faded. To obtain the maximum adsorption capacity for dyes, an FGA with a mass of $20 \mathrm{mg}$ was put into the dye solution $(50 \mathrm{ml}, 1000 \mathrm{mg} / \mathrm{l})$ for $24 \mathrm{~h}$. The maximum adsorption capacities of FGA were calculated to be 197.3, 206.2 and $596.1 \mathrm{mg} / \mathrm{g}$ for RB, MO and MB, respectively (Figure 6(d)). These results suggest that the FGA can serve as an adsorbent for organic dyes. The adsorption mechanism for dyes is as follows. After reduction of GO, the $\pi-\pi$ conjugate structure is restored between the layers; this conjugate structure interacts with the aromatic or heterocyclic structure of the dyes. ${ }^{53}$ Therefore, FGA can adsorb dyes from water.

\section{Experimental section}

\subsection{Materials}

Natural graphite powder (100 mesh), DTMS and sodium tetraborate were purchased from Shanghai Aladdin Biochemical

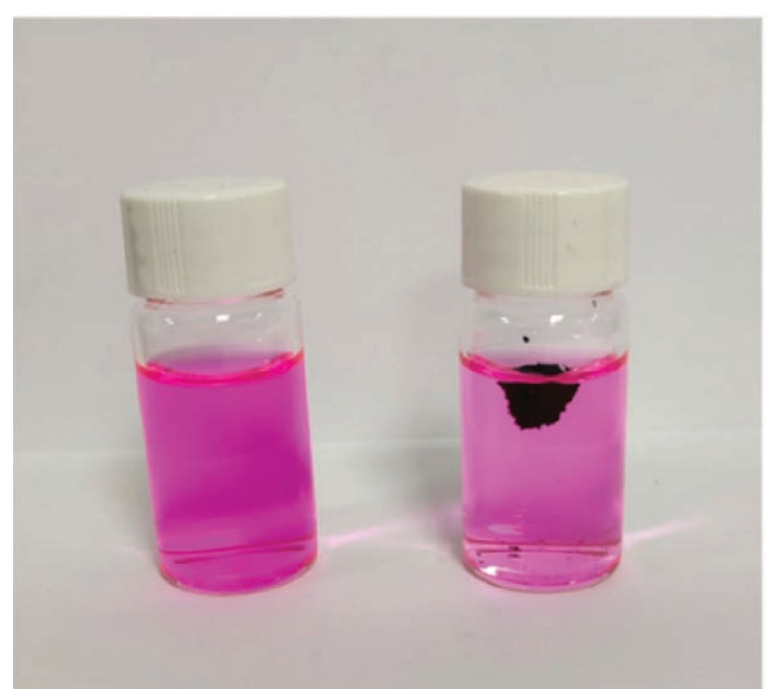

(a)

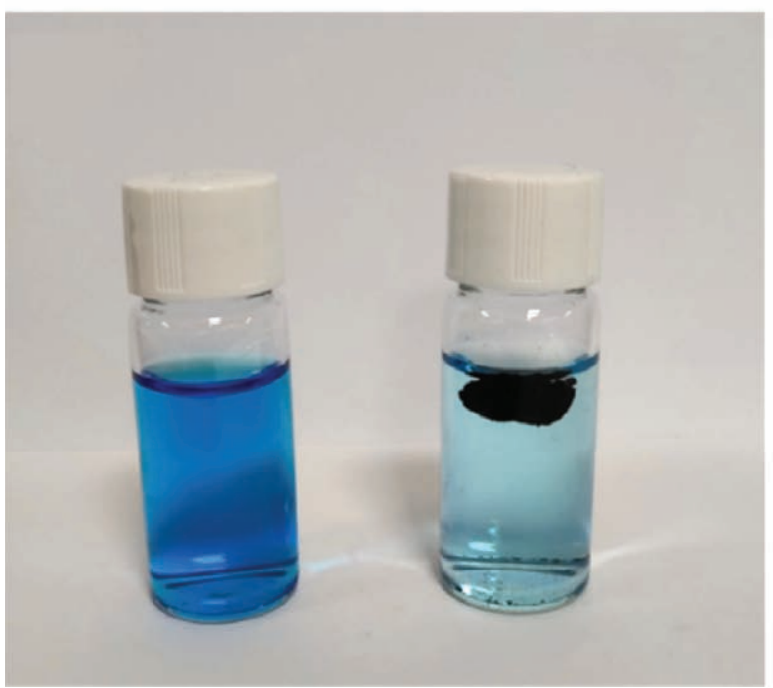

(c)

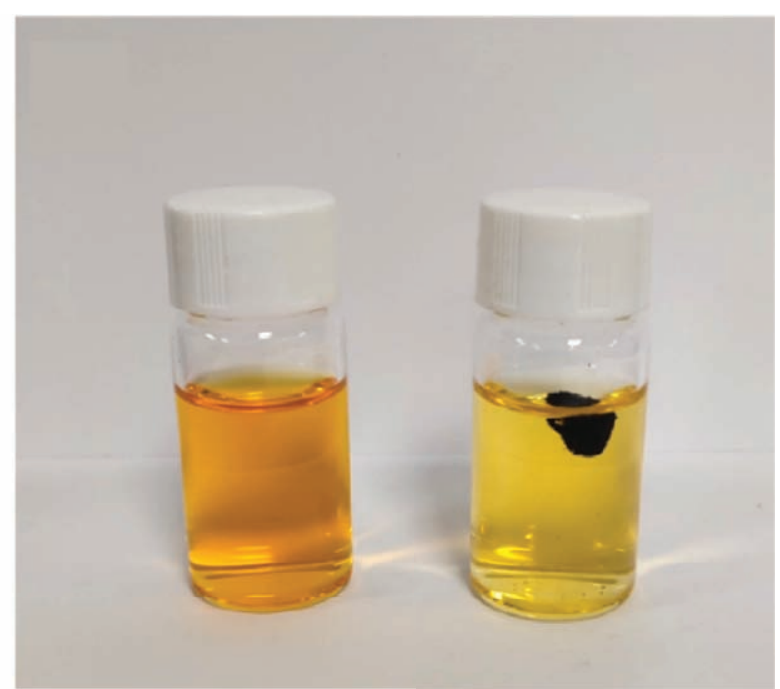

(b)

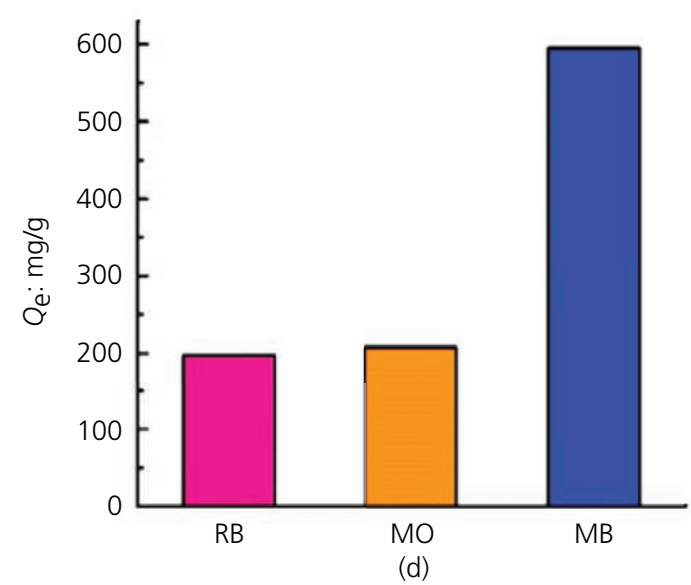

Figure 6. Dye adsorption tests of FGA. One milligram of FGA was put into (a) rhodamine B (RB), (b) MO and (c) methyl blue (MB) aqueous solutions for $24 \mathrm{~h}$. (d) Adsorption capacities of FGA for dyes 
Technology Co., Ltd. Concentrated sulfuric acid $\left(\mathrm{H}_{2} \mathrm{SO}_{4}\right)$, sodium nitrate $\left(\mathrm{NaNO}_{3}\right)$, potassium permanganate $\left(\mathrm{KMnO}_{4}\right)$, hydrogen peroxide $\left(\mathrm{H}_{2} \mathrm{O}_{2}\right)(30 \%)$, barium chloride and concentrated hydrochloric acid were provided by Tianjin Chemical Reagent Co. EDA was supplied by Tianjin Damao Chemical Reagent Factory. All reagents were used without further processing.

\subsection{Synthesis of GO}

GO was synthesized according to a modified Hummers' method as per the authors' previous report. ${ }^{54}$ The procedure involved adding graphite flakes $(5 \mathrm{~g})$ into $130 \mathrm{ml}$ concentrated sulfuric acid under constant stirring at $0^{\circ} \mathrm{C}$. Then, $2.5 \mathrm{~g}$ sodium nitrate was added into the slurry, which was agitated for another $2 \mathrm{~h}$. Afterward, $15 \mathrm{~g}$ potassium permanganate was added and stirred for $2 \mathrm{~h}$. Afterward, the suspension was heated to $35^{\circ} \mathrm{C}$ and reacted for $30 \mathrm{~min}$. After adding $230 \mathrm{ml}$ deionized water dropwise, the temperature was raised to $98^{\circ} \mathrm{C}$ and held for $30 \mathrm{~min}$. Finally, when the mixture cooled to ambient temperature, $500 \mathrm{ml}$ deionized water and $5 \mathrm{ml}$ hydrogen peroxide were added. The asprepared slurry was washed by centrifugation several times to remove residual reactant (Figure S4 in the online supplementary material).

\subsection{Synthesis of FGA}

FGA was prepared through the one-step hydrothermal method. For the typical procedure, GO dispersion at a concentration of $2 \mathrm{mg} / \mathrm{ml}$ was prepared by sonication for $3 \mathrm{~h}$. DTMS $(60 \mu \mathrm{l})$, EDA $(60 \mu \mathrm{l})$ and saturated disodium tetraborate solution $(1 \mathrm{ml})$ were added to $20 \mathrm{ml} \mathrm{GO}$ suspension. Subsequently, the mixture was transferred to a $50 \mathrm{ml}$ polytetrafluoroethylene-lined stainless-steel autoclave and kept at $120^{\circ} \mathrm{C}$ for $9 \mathrm{~h}$. The obtained FGH was put into a $20 \%$ ethanol solution for $48 \mathrm{~h}$ to remove residual reagent. Finally, the FGA was obtained by freeze-drying the FGH.

\subsection{Characterization}

SEM (SU 8010, Hitachi, Japan) was carried out to observe the morphology of aerogels at a voltage of $15 \mathrm{kV}$. The porosity and specific surface area were measured by using a Quantachrome instrument (ASIQC 050200-6, USA). The elemental maps of aerogels were recorded by XPS (Rigaku, Japan). The functional groups of aerogels were identified by FT-IR using a Thermo Fisher Scientific instrument (USA). XRD was analyzed by using a MiniFlex II X-ray diffractometer (Rigaku, Japan). Raman spectral analyses were performed with a Senterra R200-L apparatus (Bruker Optics) under a $532 \mathrm{~nm}$ excitation laser beam in the range of $500-3000 \mathrm{~cm}^{-1}$. WCA measurements were carried out by using a JC 2000C contact-angle-measuring instrument (Powereach, China). The absorbance intensity of the dye aqueous solutions was measured by using a 752 ultraviolet-visible (UV-Vis) spectrophotometer (Spectrum Instruments, Shanghai).

\subsection{Absorption capacity measurements}

The organic solvent was poured into a beaker. Then, the FGA was immersed in the organic solvent after weighing. When the absorbent reached saturation, it was weighed again. The equation is listed as follows to calculate the absorption capacity: $Q_{\mathrm{w}}$ is the adsorption capacity of FGA, $M$ is the weight after FGA has adsorbed the organic solvent and $M_{0}$ is the weight of FGA.

1. $Q_{\mathrm{w}}=\frac{M-M_{0}}{M_{0}}$

$\mathrm{RB}, \mathrm{MO}$ and $\mathrm{MB}$ were used to investigate the dye adsorption capacity of FGA. In this experiment, $20 \mathrm{mg}$ FGA was put into the aqueous dye solution $(50 \mathrm{ml}, 100 \mathrm{mg} / \mathrm{l})$; then, $1 \mathrm{ml}$ solution was taken out for detection after $24 \mathrm{~h}$. A UV-Vis spectrophotometer was used for the measurement of the dye concentration, and the maximum absorbance of each dye is $664 \mathrm{~nm}$ for $\mathrm{MB}, 464 \mathrm{~nm}$ for $\mathrm{MO}$ and $559 \mathrm{~nm}$ for RB. The following equation is listed to calculate the dye adsorption capacity: $Q_{\mathrm{e}}$ is the adsorption capacity; $m$ is the weight of FGA; $c_{0}$ and $c_{1}$ are the concentrations of the dye initially and finally, respectively; and $V$ is the volume of the aqueous dye solution.

2. $Q_{\mathrm{e}}=\frac{\left(c_{0}-c_{1}\right) \times V}{m}$

\section{Conclusion}

In conclusion, FGA with a porous structure was successfully fabricated through a one-step hydrothermal method. With the introduction of EDA reduction and DTMS modification, the FGA shows a large surface area, a structure with abundant pores and high hydrophobicity, which are beneficial for improved adsorption capability. When used as an absorbing material, the FGA exhibits highly efficient and selective adsorption of oils, organic solvents and dyes from mixtures. In addition, organic solvents adsorbed by FGA were easily collected by distillation. Moreover, FGA could be reused for organic solvent-water separation for ten cycles without affecting its oil adsorption performance, demonstrating the great reusability and durability of FGA.

\section{Acknowledgements}

This work was supported by the National Natural Science Foundation of China (21776196, 51778397), the Key R\&D projects of Shanxi Province (201803D421089) and the Shanxi Province Science Foundation for Youths (RD2000002002).

\section{REFERENCES}

1. Petrie B, Barden R and Kasprzyk-Hordern B (2015) A review on emerging contaminants in wastewaters and the environment: current knowledge, understudied areas and recommendations for future monitoring. Water Research 72: 3-27.

2. Geng JJ, Yin YW, Liang QW, Zhu ZJ and Luo HJ (2019) Polyethyleneimine cross-linked graphene oxide for removing hazardous hexavalent chromium: adsorption performance and mechanism. Chemical Engineering Journal 361: 1497-1510.

3. Kabiri S, Tran DNH, Azari S and Losic D (2015) Graphene-diatom silica aerogels for efficient removal of mercury ions from water. ACS Applied Materials \& Interfaces 7(22): 11815-11823. 
4. Kazadi Mbamba C, Lindblom E, Flores-Alsina X et al. (2019) Plantwide model-based analysis of iron dosage strategies for chemical phosphorus removal in wastewater treatment systems. Water Research 155: $12-25$.

5. Awual MR, Khraisheh M, Alharthi NH et al. (2018) Efficient detection and adsorption of cadmium(II) ions using innovative nano-composite materials. Chemical Engineering Journal 343: 118-127.

6. Liu X, Xu XT, Sun J et al. (2018) Insight into the impact of interaction between attapulgite and graphene oxide on the adsorption of U(VI). Chemical Engineering Journal 343: 217-224

7. Wang T, Dang QF, Liu CS et al. (2016) Heterotrophic nitrogen removal by a newly-isolated alkalitolerant microorganism, Serratia marcescens W5. Bioresource Technology 211: 618-627.

8. Sun ZY, Lv YK, Liu YX and Ren RP (2016) Removal of nitrogen by heterotrophic nitrification-aerobic denitrification of a novel metal resistant bacterium Cupriavidus sp. S1. Bioresource Technology 220: 142-150.

9. Dharupaneedi SP, Nataraj SK, Nadagouda M et al. (2019) Membrane-based separation of potential emerging pollutants. Separation and Purification Technology 210: 850-866.

10. Haque E, Kim J, Malgras V et al. (2018) Recent advances in graphene quantum dots: synthesis, properties, and applications. Small Methods 2(10): article 1800050 .

11. Mehta A, Mishra A, Basu S et al. (2019) Band gap tuning and surface modification of carbon dots for sustainable environmental remediation and photocatalytic hydrogen production - a review. Journal of Environmental Management 250: article 109486.

12. Reddy NL, Rao VN, Vijayakumar M et al. (2019) A review on frontiers in plasmonic nano-photocatalysts for hydrogen production. International Journal of Hydrogen Energy 44(21): 10453-10472.

13. Liew RK, Azwar E, Yek PNY et al. (2018) Microwave pyrolysis with $\mathrm{KOH} / \mathrm{NaOH}$ mixture activation: a new approach to produce micromesoporous activated carbon for textile dye adsorption. Bioresource Technology 266: 1-10.

14. Deng H, Lu JJ, Li GX, Zhang GL and Wang XG (2011) Adsorption of methylene blue on adsorbent materials produced from cotton stalk. Chemical Engineering Journal 172(1): 326-334.

15. Li ZT, Wang L, Meng J et al. (2018) Zeolite-supported nanoscale zerovalent iron: new findings on simultaneous adsorption of $\mathrm{Cd}(\mathrm{II}), \mathrm{Pb}(\mathrm{II})$, and As(III) in aqueous solution and soil. Journal of Hazardous Materials 344: 1-11.

16. Sangon S, Hunt AJ, Attard TM et al. (2018) Valorisation of waste rice straw for the production of highly effective carbon based adsorbents for dyes removal. Journal of Cleaner Production 172: 1128-1139.

17. Novais RM, Ascensao G, Tobaldi DM, Seabra MP and Labrincha JA (2018) Biomass fly ash geopolymer monoliths for effective methylene blue removal from wastewaters. Journal of Cleaner Production 171: 783-794.

18. Lee C, Wei XD, Kysar JW and Hone J (2008) Measurement of the elastic properties and intrinsic strength of monolayer graphene. Science 321(5887): 385-388.

19. Balandin AA, Ghosh S, Bao WZ et al. (2008) Superior thermal conductivity of single-layer graphene. Nano Letters 8(3): 902-907.

20. Li XL, Zhang GY, Bai XD et al. (2008) Highly conducting graphene sheets and Langmuir-Blodgett films. Nature Nanotechnology 3(9): 538-542.

21. Shetti NP, Malode SJ, Nayak DS et al. (2019) A novel biosensor based on graphene oxide-nanoclay hybrid electrode for the detection of Theophylline for healthcare applications. Microchemical Journal 149: article 103985 .

22. Kumar S, Bukkitgar SD, Singh S et al. (2019) Electrochemical sensors and biosensors based on graphene functionalized with metal oxide nanostructures for healthcare applications. ChemistrySelect 4(18): 5322-5337.

23. Murat C, Kakarla RR and Alonso-Marroquin F (2017) Advanced electrochemical energy storage supercapacitors based on the flexible carbon fiber fabric-coated with uniform coral-like $\mathrm{MnO}_{2}$ structured electrodes. Chemical Engineering Journal 309: 151-158.

24. Mishra A, Mehta A, Basu S et al. (2019) Graphitic carbon nitride (g$\mathrm{C}_{3} \mathrm{~N}_{4}$ )-based metal-free photocatalysts for water splitting: a review. Carbon 149: 693-721.

25. Reddy KR, Reddy CV, Nadagouda MN et al. (2019) Polymeric graphitic carbon nitride $\left(\mathrm{g}-\mathrm{C}_{3} \mathrm{~N}_{4}\right)$-based semiconducting nanostructured materials: synthesis methods, properties and photocatalytic applications. Journal of Environmental Management 238: 25-40.

26. Haque E, Yamauchi Y, Malgras V et al. (2018) Nanoarchitectured graphene-organic frameworks (GOFs): synthetic strategies, properties, and applications. Chemistry - an Asian Journal 13(23): 3561-3574.

27. Xu X, Zhang QQ, Yu YK et al. (2016) Naturally dried graphene aerogels with superelasticity and tunable Poisson's ratio. Advanced Materials 28(41): 9223-9230.

28. Chen K, Shi L, Zhang Y and Liu Z (2018) Scalable chemical-vapourdeposition growth of three-dimensional graphene materials towards energy-related applications. Chemical Society Reviews 47(9): 3018-3036.

29. Choi YS, Yeo CS, Kim SJ et al. (2019) Multifunctional reduced graphene oxide-CVD graphene core-shell fibers. Nanoscale 11(26): 12637-12642

30. Mi HY, Jing X, Politowicz AL et al. (2018) Highly compressible ultralight anisotropic cellulose/graphene aerogel fabricated by bidirectional freeze drying for selective oil absorption. Carbon 132: 199-209.

31. Zhu C, Liu TY, Qian F et al. (2016) Supercapacitors based on threedimensional hierarchical graphene aerogels with periodic macropores. Nano Letters 16(6): 3448-3456.

32. Chen C, Li F, Zhang Y et al. (2018) Compressive, ultralight and fireresistant lignin-modified graphene aerogels as recyclable absorbents for oil and organic solvents. Chemical Engineering Journal 350: 173-180.

33. Hong JY, Sohn EH, Park S and Park HS (2015) Highly-efficient and recyclable oil absorbing performance of functionalized graphene aerogel. Chemical Engineering Journal 269: 229-235.

34. Chen C, Zhu XY and Chen BL (2018) Covalently cross-linked graphene oxide aerogel with stable structure for high-efficiency water purification. Chemical Engineering Journal 354: 896-904.

35. Salon MCB, Bayle PA, Abdelmouleh M, Boufi S and Belgacem MN (2008) Kinetics of hydrolysis and self-condensation reactions of silanes by NMR spectroscopy. Colloids and Surfaces A: Physicochemical and Engineering Aspects 312(2-3): 83-91.

36. Guan LZ, Gao JF, Pei YB et al. (2016) Silane bonded graphene aerogels with tunable functionality and reversible compressibility. Carbon 107: 573-582.

37. Basavarajappa PS, Seethya BNH, Ganganagappa N, Eshwaraswamy KB and Kakarla RR (2018) Enhanced photocatalytic activity and biosensing of gadolinium substituted $\mathrm{BiFeO}_{3}$ nanoparticles. ChemistrySelect 3(31): 9025-9033.

38. Tavangar T, Karimi M, Rezakazemi M, Reddy KR and Aminabhavi TM (2020) Textile waste, dyes/inorganic salts separation of cerium oxideloaded loose nanofiltration polyethersulfone membranes. Chemical Engineering Journal 385: article 123787.

39. Kim SW, Kim HK, Lee K et al. (2019) Studying the reduction of graphene oxide with magnetic measurements. Carbon 142: 373-378.

40. Reddy KR, Gomes VG and Hassan M (2014) Carbon functionalized $\mathrm{TiO}_{2}$ nanofibers for high efficiency photocatalysis. Materials Research Express 1(1): article 015012.

41. Geng J, Yin Y, Liang Q, Zhu Z and Luo H (2019) Polyethyleneimine cross-linked graphene oxide for removing hazardous hexavalent chromium: adsorption performance and mechanism. Chemical Engineering Journal 361: 1497-1510.

42. Xiong DB, Li XF, Bai ZM et al. (2017) Superior cathode performance of nitrogen-doped graphene frameworks for lithium ion batteries. ACS Applied Materials \& Interfaces 9(12): 10643-10651. 
43. Li Y, Cui WQ, Liu L et al. (2016) Removal of Cr(VI) by 3D $\mathrm{TiO}_{2}$-graphene hydrogel via adsorption enriched with photocatalytic reduction. Applied Catalysis B: Environmental 199: 412-423.

44. Reddy KR, Sin BC, Ryu KS, Noh J and Lee Y (2009) In situ selforganization of carbon black-polyaniline composites from nanospheres to nanorods: synthesis, morphology, structure and electrical conductivity. Synthetic Metals 159(19-20): 1934-1939.

45. Reddy KR, Nakata K, Ochiai T et al. (2010) Nanofibrous $\mathrm{TiO}_{2}$-core/ conjugated polymer-sheath composites: synthesis, structural properties and photocatalytic activity. Journal of Nanoscience and Nanotechnology 10(12): 7951-7957.

46. Li GF, Wang X, Tao L et al. (2015) Cross-linked graphene membrane for high-performance organics separation of emulsions. Journal of Membrane Science 495: 439-444.

47. Zhang Q, Xu X, Li H, Xiong G, Hu H and Fisher TS (2015) Mechanically robust honeycomb graphene aerogel multifunctional polymer composites. Carbon 93: 659-670.

48. Reddy NR, Bhargav U, Kumari MM et al. (2020) Highly efficient solar light-driven photocatalytic hydrogen production over $\mathrm{Cu} / \mathrm{FCNTs}$ titania quantum dots-based heterostructures. Journal of Environmental Management 254: article 109747.
49. Han Z, Tang ZH, Li P et al. (2013) Ammonia solution strengthened three-dimensional macro-porous graphene aerogel. Nanoscale 5(12): 5462-5467.

50. Xiao JL, Lv WY, Song YH and Zheng Q (2018) Graphene/nanofiber aerogels: performance regulation towards multiple applications in dye adsorption and oil/water separation. Chemical Engineering Journal 338: $202-210$.

51. Ge J, Zhao HY, Zhu HW et al. (2016) Advanced sorbents for oil-spill cleanup: recent advances and future perspectives. Advanced Materials 28(47): 10459-10490.

52. Feng $X$ and Jiang $L$ (2006) Design and creation of superwetting/ antiwetting surfaces. Advanced Materials 18(23): 3063-3078.

53. Xiao JL, Zhang JF, Lv WY, Song YH and Zheng Q (2017) Multifunctional graphene/poly(vinyl alcohol) aerogels: in situ hydrothermal preparation and applications in broad-spectrum adsorption for dyes and oils. Carbon 123: 354-363.

54. Ren RP, Li W and Lv YK (2017) A robust, superhydrophobic graphene aerogel as a recyclable sorbent for oils and organic solvents at various temperatures. Journal of Colloid and Interface Science 500: 63-68.

\section{How can you contribute?}

To discuss this paper, please submit up to 500 words to the journal office at journals@ice.org.uk. Your contribution will be forwarded to the author(s) for a reply and, if considered appropriate by the editor-in-chief, it will be published as a discussion in a future issue of the journal.

ICE Science journals rely entirely on contributions from the field of materials science and engineering. Information about how to submit your paper online is available at www.icevirtuallibrary.com/page/authors, where you will also find detailed author guidelines. 